\title{
Microstructural Understanding of the Length- and Stiffness- Dependent Shear Thinning in Semidilute Colloidal Rods
}

\author{
Christian Lang, ${ }^{*} \dagger \odot$ Joachim Kohlbrecher, ${ }^{*}$, Lionel Porcar, $*, \|^{*}$ Aurel Radulescu, ${ }^{* \dagger}$ Karin Sellinghoff, ${ }^{*}, *$ \\ Jan Karel George Dhont, ${ }^{*}+, \perp$ and Minne Paul Lettinga*,+\# \\ $\dagger$ Jülich Centre for Neutron Science and ${ }^{\ddagger}$ Institute of Complex Systems 3, Forschungszentrum Jülich, 52425 Jülich, Germany \\ ${ }^{\S}$ Laboratory of Neutron Scattering and Imaging, Paul Scherrer Institute, Villigen 5232, Switzerland \\ "Institut Laue-Langevin, Grenoble 38000, France \\ ${ }^{\perp}$ Experimental Physics of Soft Matter, Heinrich Heine Universität Düsseldorf, 40225 Düsseldorf, Germany \\ ${ }^{\#}$ Laboratory of Soft Matter and Biophysics, Katholieke Universiteit Leuven, 3000 Leuven, Belgium
}
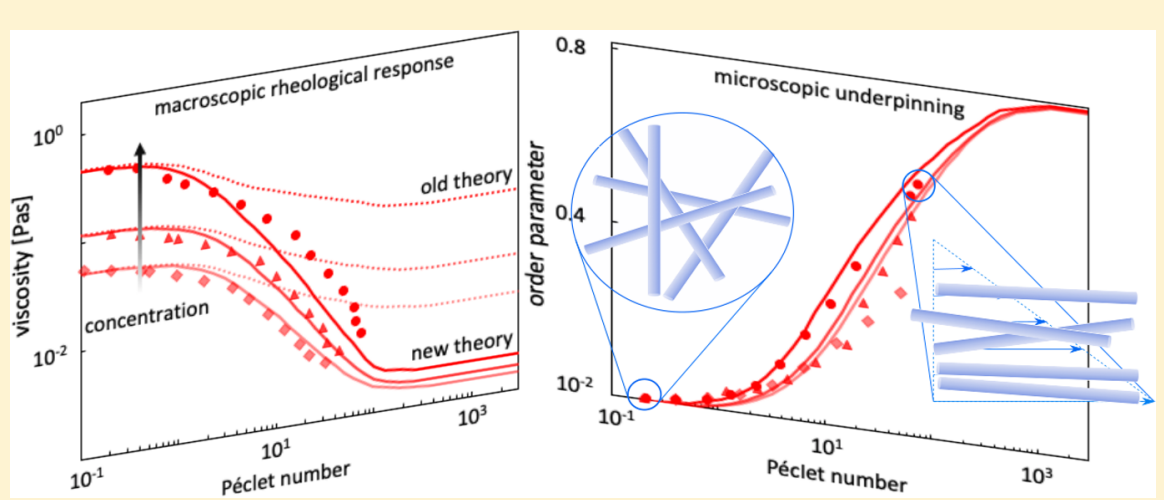

ABSTRACT: Complex fluids containing low concentrations of slender colloidal rods can display a high viscosity, while little flow is needed to thin the fluid. This feature makes slender rods essential constituents in industrial applications and biology. Though this behavior strongly depends on the rod-length, so far no direct relation could be identified. We employ a library of filamentous viruses to study the effect of rod size and flexibility on the zero-shear viscosity and shear-thinning behavior. Rheology and small-angle neutron scattering data are compared to a revised version of the standard theory for ideally stiff rods, which incorporates a complete shear-induced dilation of the confinement. While the earlier predicted length-independent prefactor of the restricted rotational diffusion coefficient is confirmed by varying the length and concentration of the rods, the revised theory correctly predicts the shear-thinning behavior as well as the underlying orientational order. These results can be directly applied to understand the manifold systems based on rodlike colloids and design new materials.

\section{INTRODUCTION}

Rodlike colloids are present in a broad diversity of daily-life products, like polymeric materials, ${ }^{1-6}$ food-, and dairy products, ${ }^{7-10}$ and also play an important role in living organisms. ${ }^{11-14}$ The reason is that at very low volume fractions, rods will yield a fluid extremely viscous, while little flow is needed to thin the fluid. ${ }^{15-19}$ Though theory predicts that the mechanical response of fluids containing rodlike colloids is hugely affected by the particle length, flexibility, and concentration, the lack of model systems has impeded a full understanding of the shear-thinning behavior.

The goal of this article is to identify how the rodlike particles' aspect ratio, flexibility, and microstructural order affect shear thinning. To this end, it is important to develop a suitable model system for which a controlled variation of aspect ratio and flexibility is feasible. In particular, it is important to have access to monodisperse systems, since a variation in particle size and flexibility would blur the differences between the various systems. A very promising way of producing such monodisperse systems is through bioengineering of rodlike viruses. ${ }^{20}$ We use rodlike bacteriophages from the lambda class, ${ }^{21}$ namely fd wild type, fdY21M, M13k07, and Pf1 viruses, as these species span a range of $0.9-2 \mu \mathrm{m}$ in length as well as a range of $1.2-9.9 \mu \mathrm{m}$ in persistence length, while the thickness for all these viruses is close to roughly $7 \mathrm{~nm}$. The effective rod thickness that determines the range of inter-rod interactions is modified by surface coating ${ }^{22}$ and changing the ionic strength of the suspending medium. ${ }^{23}$ These systems have proven to be ideally suited to study the flow behavior of rods. ${ }^{18,24-28}$ It is equally important to perform experiments that can relate the flowinduced orientational ordering with the rheological behavior of

Received: July 31, 2019

Revised: November 20, 2019

Published: December 3, 2019 
the different systems. In situ small-angle neutron scattering in combination with rheology (rheo-SANS) is particularly suited to obtain this relation ${ }^{26,27,29}$ because of the high contrast of this technique. Moreover, performing the scattering experiments yields the full 3D orientational distribution. ${ }^{26,30,31}$ Thus, the core of this article is to determine the flow curves for all of the systems at varying concentrations and measuring the viscosity and orientational ordering as a function of the applied shear rate with rheo-SANS. This allows us to relate the structural and mechanical response.

The experimental results require an extension of the existing theory with a direct relation between the restricted motion of the rods and the degree of flow-induced alignment. To understand this relation, we start from the observation that the excluded volume for other rods resulting from adding a slender rod to a fluid strongly increases with the concentration of the rods. This excluded volume causes a cascade of phase transitions with increasing concentration, depending on the rod aspect ratio, ${ }^{32,33}$ the flexibility, ${ }^{34,35}$ and the interactions between the rodlike particles. ${ }^{36}$ Similarly, these single-particle properties affect the flow behavior in the disordered semidilute isotropic phase, where the number density $\nu=N / V_{\text {tot }}$ is already much larger than the overlap number density $\nu^{*} \sim 1$ / $L^{3}$ (with $N$ the number of particles, $L$ the particle length, and $V_{\text {tot }}$ the overall sample volume). ${ }^{37-44}$ As a result, the rotational dynamics of particles is strongly reduced, leading to a strong shear-thinning behavior, ${ }^{26,45,46}$ where the viscosity strongly reduces with increasing shear rate, while the viscosity at zeroshear strongly increases with concentration. The reduced rotational diffusion is the base of the seminal theory for ideally stiff rods under shear flow, as proposed by Doi, Edwards, and Kuzuu, ${ }^{38,47-49}$ where this aspect is taken into account. However, a full dilation of the confining tube in strong applied flow fields has not been realized. We will show how an extended version of the theory takes this dilation into account, thus describing the complete shear-thinning behavior in dispersions of colloidal rods.

This article is structured as follows. We begin with a discussion of a theory for the shear-thinning behavior and shear-induced nematiclike order in rodlike colloidal systems, followed by a description of the rheo-SANS procedures as well as the bioengineered viruses used in this study. The experimental findings are detailed in Section 4, with a concluding discussion in Section 5.

\section{THEORY}

A theory for the response of suspensions of uniaxial, stiff, and monodisperse rodlike particles to shear flow has been put forward by Doi, Edwards, and Kuzuu, which we will refer to as the Doi-Edwards-Kuzuu (DEK) theory. ${ }^{38,47-49}$ A similar approach has been proposed by Hess. ${ }^{37}$ A microscopic foundation of the DEK theory is discussed in ref44, where the following Fokker-Planck type equation of motion for the probability density function $\psi(\hat{\mathbf{u}} ; t)$ of the orientation $\hat{\mathbf{u}}$ of a test rod is derived from the $\mathrm{N}$-particle Smoluchowski equation $^{50}$

$$
\frac{\partial \psi}{\partial t}=D_{\mathrm{r}}^{0} \hat{\mathcal{R}} \cdot[\hat{\mathcal{R}} \psi-\beta \psi \overline{\mathbf{T}}]-\hat{\mathcal{R}} \cdot[\psi \hat{\mathbf{u}} \times(\boldsymbol{\Gamma} \cdot \hat{\mathbf{u}})]
$$

where $D_{\mathrm{r}}^{0}$ is the rotational diffusion coefficient of a freely rotating rod, $\Gamma$ is the velocity-gradient tensor, and $\hat{\mathcal{R}}(\cdot)=\hat{\mathbf{u}} \times \nabla_{\hat{\mathbf{u}}}(\cdot)$ is the rotation operator, with $\nabla_{\hat{\mathbf{u}}}$ the gradient operator with respect to the Cartesian coordinates of $\hat{\mathbf{u}}$. Furthermore, $\overline{\mathbf{T}}$ is the effective torque acting on the test particle due to the presence of the other rods

$$
\overline{\mathbf{T}}=-\nu \int \mathrm{d} \mathbf{R} \oint \mathrm{d} \hat{\mathbf{u}}^{\prime} \psi\left(\hat{\mathbf{u}}^{\prime} ; t\right) g\left(\mathbf{R}, \hat{\mathbf{u}}, \hat{\mathbf{u}}^{\prime} ; t\right) \hat{\mathcal{R}} \mathcal{V}\left(\mathbf{R}, \hat{\mathbf{u}}, \hat{\mathbf{u}}^{\prime}\right)
$$

where $\nu$ is the number density of rods, $\mathbf{R}$ is the distance between the centers of two rods, $\mathcal{V}\left(\mathbf{R}, \hat{\mathbf{u}}, \hat{\mathbf{u}}^{\prime}\right)$ is the pairinteraction potential, and $g\left(\mathbf{R}, \hat{\mathbf{u}}, \hat{\mathbf{u}}^{\prime} ; t\right)$ is the pair-correlation function. The integral in eq 2 with respect to $\hat{\mathbf{u}}^{\prime}$ ranges over the unit spherical surface. The torque in eq 2 is the torque $-\hat{\mathcal{R}} \mathcal{V}$ on the test rod due to the interaction with a neighboring rod, averaged with respect to the orientation and position of the latter. The effective torque is a function of concentration and shear rate through the pair-correlation function.

Within the DEK theory, instead of the effective torque in eq 2 , the torque is assumed to take the form $-\hat{\mathcal{R}} \mathcal{V}_{\mathrm{mf}}$, where $\mathcal{V}_{\mathrm{mf}}$ is the mean-field Maier-Saupe potential $\sim \mathbf{S}: \hat{\mathbf{u}} \hat{\mathbf{u}}$, where $\mathbf{S}=\langle\hat{\mathbf{u}}$ $\hat{\mathbf{u}}\rangle$ is the orientational order parameter tensor. This mean-field potential can be derived from eq 2 by using the pair-correlation function $g=\exp \{-\beta \mathcal{V}\}$ for long and thin rods with (effective) hard-core interactions, which neglects the effect of shear flow on inter-rod correlations. ${ }^{44}$ In the DEK approach and its microscopic foundation in ref 44, hydrodynamic interactions between the rods are neglected, which is a good approximation for long and thin rods.

The problem that the microscopic approach faces is that the analytical computation of an accurate expression for the paircorrelation function, including the effect of shear flow and multiple-rod interactions, is not feasible. In the approach in ref 44 , the pair-correlation function is simply approximated by the two-particle equilibrium pair-correlation function $g=\exp \{-\beta \mathcal{V}\}$ in eqs land 2 , leading to the following equation of motion for $S$, which is valid up to the third order in the orientational order parameter tensor

$$
\begin{aligned}
\frac{\mathrm{d} \mathbf{S}}{\mathrm{d} t} & =-6 D_{\mathrm{r}}^{0}\left[\mathbf{S}-\frac{1}{3} \mathbf{I}+\frac{L}{d} \varphi\left(\mathbf{S}^{(4)}: \mathbf{S}-\mathbf{S} \cdot \mathbf{S}\right)\right] \\
& +\dot{\gamma}\left[\hat{\boldsymbol{\Gamma}} \cdot \mathbf{S}+\mathbf{S} \cdot \hat{\boldsymbol{\Gamma}}^{T}-2 \mathbf{S}^{(4)}: \hat{\mathbf{E}}\right]
\end{aligned}
$$

where $\dot{\gamma}$ is the shear rate, $\mathbf{I}$ is the identity tensor, $\hat{\Gamma}=\Gamma / \dot{\gamma}$ and, similarly, $\hat{\mathbf{E}}=\mathbf{E} / \dot{\gamma}$ where $\mathbf{E}$ is the symmetric part of the velocity-gradient tensor, $L$ is the length, $d$ is the diameter of the rods, and $\varphi=\nu L d^{2} \pi / 4$ is the volume fraction of rods. Furthermore, $\mathbf{S}^{(4)}=\langle\hat{\mathbf{u}} \hat{u} \hat{u} \hat{u}\rangle$, for which a closure relation will be discussed below. This approximation accounts only for simultaneous interactions of just two rods, which is, as shown by Onsager, ${ }^{32,51}$ exact for very long and thin rods in equilibrium up to concentrations well within the nematic state. Under flow conditions, however, inter-rod correlations are affected by dynamical events which involve topological constraints where multiple-rod interactions are essential. As in the DEK theory, we will account for such topological constraints by replacing the bare rotational diffusion coefficient $D_{\mathrm{r}}^{0}$ by an effective diffusion coefficient $D_{\mathrm{r}}^{\text {eff }}$ that accounts for such constraints. At first sight, one might think that $D_{\mathrm{r}}^{0}$ should only be replaced by the effective diffusion coefficient in the term $\sim(L / d) \varphi$ in eq 3 that involves the pair-correlation function. This leads, however, to a thermodynamic inconsistency. ${ }^{52}$ We therefore replace the bare diffusion coefficient in eq 3 also for the ideal relaxation contribution $\sim(S-(1 / 3)$ 
$\hat{\mathbf{I}})$. The interpretation for such an overall replacement is that each rod is embedded in an effective medium, which also affects the otherwise ideal relaxation contribution. Replacing the bare diffusion coefficient $D_{\mathrm{r}}^{0}$ by the effective diffusion coefficient $D_{\mathrm{r}}^{\text {eff }}$ leads to the following dimensionless equation of motion

$$
\begin{aligned}
& \frac{\mathrm{d} \mathbf{S}}{\mathrm{d} \tau}=-\left(\mathbf{S}-\frac{1}{3} \mathbf{I}\right)-\frac{L}{d} \varphi\left(\mathbf{S}^{(4)}: \mathbf{S}-\mathbf{S} \cdot \mathbf{S}\right) \\
& +\frac{1}{6} \operatorname{Pe}\left[\hat{\boldsymbol{\Gamma}} \cdot \mathbf{S}+\mathbf{S} \cdot \hat{\boldsymbol{\Gamma}}^{T}-2 \mathbf{S}^{(4)}: \hat{\mathbf{E}}\right]
\end{aligned}
$$

where the dimensionless time $\tau=6 D_{\mathrm{r}}^{\text {eff }} t$ is introduced and where the dimensionless effective rotational Peclet number is given by

$$
P e=\dot{\gamma} / D_{\mathrm{r}}^{\mathrm{eff}}
$$

This is the fundamental equation of motion on the basis of which the experiments concerning shear-induced alignment will be discussed. To this end, however, the higher-order average $\boldsymbol{S}^{(4)}$ must be expressed in terms of $\boldsymbol{S}$. Within the DEK theory, a simple quadratic factorization closure relation is used. Here, we will employ the more accurate closure relation that has been proposed in ref 44

$$
\begin{aligned}
\mathbf{S}^{(4)}: \mathbf{M}= & \frac{1}{5}\{\mathbf{S} \cdot \overline{\mathbf{M}}+\overline{\mathbf{M}} \cdot \mathbf{S}-\mathbf{S} \cdot \mathbf{S} \cdot \overline{\mathbf{M}}-\overline{\mathbf{M}} \cdot \mathbf{S} \cdot \mathbf{S}+2 \mathbf{S} \cdot \overline{\mathbf{M}} \cdot \mathbf{S} \\
& +3 \mathbf{S ~ S :} \overline{\mathbf{M}}\}
\end{aligned}
$$

where

$$
\overline{\mathbf{M}}=\frac{1}{2}\left[\mathbf{M}+\mathbf{M}^{\mathbf{T}}\right]
$$

is the symmetric part of the tensor $\mathbf{M}$. In the construction of this closure relation, the quantity $\mathbf{S}^{(4)}: \mathbf{M}$ is assumed to be a linear combination of all possible terms that are linear or second order in $\mathbf{S}$. The coefficients of these terms in such a linear combination are then determined from the requirements that the closure should be valid in the isotropic and perfectly aligned state, for which $\mathbf{S}^{(4)}$ is known, and from the fact that $\mathbf{S}^{(4)}: \mathbf{M}$ is symmetric and its trace is equal to $\mathbf{S}: \mathbf{M}$. Note that various more accurate closure schemes exist for different nonequilibrium situations. ${ }^{53}$

Within the realm of a tube model for rotational diffusion in simple shear flow, Doi proposed an expression for the effective diffusion coefficient, which is valid in the semidilute regime and for moderate degrees of rod alignment. ${ }^{47}$ In the present article, we present experiments up to very high shear rates, where there is a high degree of alignment. For such high shear rates, the tube model no longer applies, and the rotational diffusion coefficient is essentially equal to $D_{\mathrm{r}}^{0}$. We thus need to interpolate between Doi's expression and $D_{\mathrm{r}}^{0}$. Such an interpolation is established by the following expression

$$
D_{\mathrm{r}}^{\mathrm{eff}}=\frac{D_{\mathrm{r}}^{0}}{1+\frac{1}{c}\left[\frac{5}{4} \nu L^{3}\left(1-\frac{3}{5} \mathbf{S}: \mathbf{S}-\frac{2}{5}(\mathbf{S}: \mathbf{S})^{2}\right)\right]^{2}}
$$

This expression essentially coincides with the original Doi expression in the semidilute regime and for moderate shear rates and becomes equal to $D_{\mathrm{r}}^{0}$ for low concentrations, ${ }^{54}$ as well as high shear rates. The coefficient $c \approx 1.3 \times 10^{3}$ was obtained by Teraoka et al. $^{55}$ and by Tao et al. ${ }^{56}$ using Brownian dynamics simulations. The concentration dependence of the effective diffusion coefficient in the absence of shear flow found in ref 56 is in agreement with eq 8 over the entire concentration range. In Section 4, we discuss how $c$ can be determined experimentally.

Within the approach in ref 44, the relation between the deviatoric part of the stress tensor $\sum_{D}$ and the orientational order parameter tensor is based on a microscopic expression that is valid for rigid colloids as derived in ref 57. Using $D_{\mathrm{r}}^{0}=$ $3 k_{\mathrm{B}} T \ln \{L / d\} / \pi \eta_{\mathrm{s}} L^{3}$, we find

$$
\begin{aligned}
& \sum_{D}=2 \eta_{\mathrm{s}} \dot{\gamma} \hat{\mathbf{E}}+3 \nu k_{\mathrm{B}} T\left\{\left[\mathbf{S}-\frac{1}{3} \mathbf{I}+\frac{L}{d} \varphi\left(\mathbf{S}^{(4)}: \mathbf{S}-\mathbf{S} \cdot \mathbf{S}\right)\right]\right. \\
& \left.+\frac{1}{6} P e^{0}\left[\mathbf{S}^{(4)}: \hat{\mathbf{E}}-\frac{1}{3} \mathbf{I} \mathbf{S}: \hat{\mathbf{E}}\right]\right\}
\end{aligned}
$$

where $\eta_{\mathrm{s}}$ is the shear viscosity of the solvent and

$$
P e^{0}=\dot{\gamma} / D_{\mathrm{r}}^{0}
$$

is the bare Peclet number.

Note that the effective Peclet number does not appear in this expression. Although the prefactor of the last term in eq 9 contains the functional form of the inverse bare diffusion coefficient, it cannot be interpreted in terms of a diffusion coefficient, also because it is not related to the only contribution $\sim(L / d) \varphi$ that is connected to the paircorrelation function. The stress in the present approach is therefore only indirectly affected by entanglements through the dependence of $\mathbf{S}$ on the effective diffusion coefficient. A similar expression for the stress tensor, based on thermodynamic arguments, has been put forward within the DEK theory.

The zero-shear viscosity can be obtained from eqs 4 and 9 by expanding $\mathbf{S}$ up to first order in the shear rate. From the stationary equation of motion (eq 4 ), it is found that

$$
\mathbf{S}=\frac{1}{3} \hat{\mathbf{I}}+\frac{1}{15} \frac{\dot{\gamma}}{\tilde{D}_{\mathrm{r}}^{\text {eff }}} \hat{\mathbf{E}}+O\left(P e^{2}\right)
$$

where a second effective diffusion $\tilde{D}_{\mathrm{r}}^{\text {eff }}$ is introduced that also accounts for the slowing down of orientational diffusion due to the proximity of the isotropic-nematic spinodal, which is given by

$$
\tilde{D}_{\mathrm{r}}^{\text {eff }}=\left(1-\frac{L}{5 d} \varphi\right) D_{\mathrm{r}}^{\mathrm{eff}}
$$

On substitution into eq 9 and again linearisation with respect to the shear rate, it is readily found that the zero-shear viscosity $\eta_{0}$ is given by $^{47}$

$$
\eta_{0}=\eta_{\mathrm{s}}+\nu k_{\mathrm{B}} T\left[\frac{1}{30} \frac{1}{D_{\mathrm{r}}^{0}}+\frac{1}{10} \frac{1}{D_{\mathrm{r}}^{\text {eff }}}\right]
$$

The first term in the square brackets is a viscous contribution that arises from the expression (9) for the stress tensor, while the second contribution is an elastic contribution, as it arises from correlations between the rods as captured by the equation of motion (eq 4) for the order parameter tensor. In the semidilute regime and for the small shear rate under consideration here, eq 8 reduces to the original Doi's expression. Substituting $\mathbf{S}=(1 / 3) \hat{\mathbf{I}}$, the appropriate expression for the rotational diffusion coefficient that is to be used in the above expression for the zero-shear viscosity is therefore $D_{\mathrm{r}}^{\text {eff }}=$ 
$c D_{\mathrm{r}}^{0} / \nu^{2} L^{6}$. In the semidilute regime, where $D_{\mathrm{r}}^{0} \gg D_{r}^{\text {eff }}$, the first contribution in eq 13 is of minor importance.

eEquations 4,6, 8,9, and 13 present a full theoretical prediction for the concentration- and shear-rate-dependent orientational order and viscosity for stiff rods that will be used in Section 4 in comparison to experimental results.

\section{EXPERIMENTS AND MATERIALS}

Experiments. Rheo-SANS studies were carried out at the SINQ spallation source in Villigen, Switzerland, the Heinz Maier-Leibnitz Zentrum in Garching, Germany, and the Institut Laue-Langevin in Grenoble, France. An Anton Paar MCR 501 rheometer (Anton Paar, Graz, Austria) was mounted in the SANS-1 and KWS-2 neutron beams for simultaneous measurements of shear stress and orientational ordering in the velocity-vorticity plane. The used sample environment was a Couette cell with a gap size of $1 \mathrm{~mm}$ and with an inner-cell radius of $48 \mathrm{~mm}$. Since the Anton Paar MCR 501 is a stresscontrolled rheometer, all rheological measurements were repeated with a strain-controlled ARES LS rheometer (TA Instruments, New Castle, PA). For measurements in the velocity-gradient plane, a shear cell was mounted at the D22 diffractometer. For measurements at extremely low shear rates, of the order $\dot{\gamma} \sim 10^{-5} \mathrm{~s}^{-1}$, the shear cell was equipped with a state-of-the-art brushless ec-motor and a gearbox transducer. All measurements were performed at $25{ }^{\circ} \mathrm{C}$ with a detector distance of $4.6 \mathrm{~m}$ and a wavelength of $0.6 \pm 0.1 \mathrm{~nm}$ at SANS1 , a detector distance of $5.6 \mathrm{~m}$, a wavelength of $0.6 \pm 0.1 \mathrm{~nm}$, and an aperture size of $3 \times 0.15 \mathrm{~mm}$ at D22, and a detector distance of $8 \mathrm{~m}$ and a wavelength of $0.5 \pm 0.1 \mathrm{~nm}$ at KWS-2. From the available $q$ range at the different instruments, we selected a small subset of $3.2 \times$ $10^{-2}$ to $4.6 \times 10^{-2} \AA$ (in the Porod regime) for our analysis. In this regime, the different reduction schemes for extracting the orientational distribution of rods from two-dimensional scattering patterns by Hayter and Penfold ${ }^{58}$ and Maier and Saupe ${ }^{59}$ give similar results.

We find the projections of the orientational distribution in the flowvorticity (1-3) plane from the intensity profiles as a function of $\phi$ and in the flow-gradient (1-2) plane from the intensity profiles as a function of $\alpha$, see Figure 1, and by fitting these profiles with a MaierSaupe type ${ }^{59}$ of orientational distribution function, $f(\phi)=I_{0}$ exp$\left(\tilde{c} P_{2}\left(\phi-\phi_{\max }\right)-1\right)$. Here, $I_{0}$ is the amplitude, $\tilde{c}$ the width, and $\phi_{\max }$

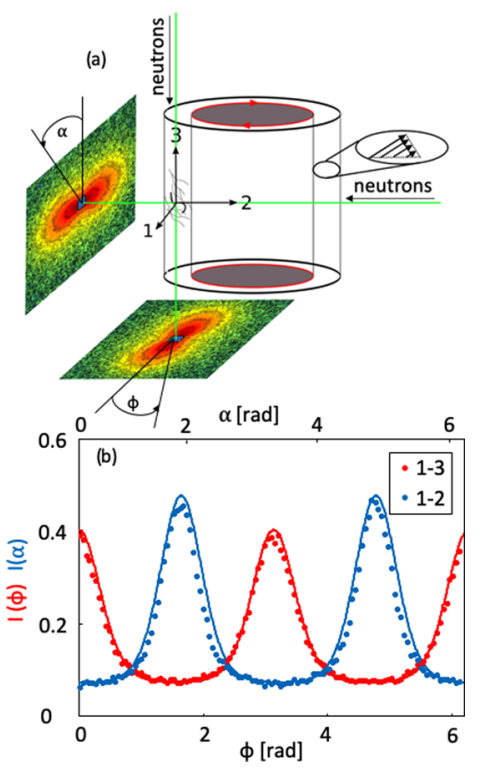

Figure 1. (a) Sketch of the rheo-SANS setup (Couette cell). (b) Intensity measured in the velocity-vorticity (1-3) and velocitygradient (1-2) plane as a function of the azimuthal angle for fdY21M at a concentration of $10.6 \mathrm{mg} / \mathrm{mL}$ with $\dot{\gamma}=64 \mathrm{~s}^{-1}$. Lines are fitted Maier-Saupe distributions. the tilt angle of the projected nematic director with respect to the direction of flow. From this distribution, we find the projected order parameters as $\left\langle P_{2}\right\rangle=\int_{0}^{1} \mathrm{~d} \cos (\phi) f(\phi) P_{2}\left(\phi-\phi_{\max }\right) / \int_{0}^{1} \mathrm{~d} \cos (\phi) f(\phi)$. The identical relations can be written for $\phi$ replaced by the angle $\alpha$. From these projections, the largest eigenvalues, $\lambda_{1}(\phi)$ and $\lambda_{1}(\alpha)$, of the projections of the traceless orientation tensor $\tilde{\mathbf{Q}}$ are found as $\lambda_{1}(\phi)=\left(2\left\langle P_{2}(\phi)\right\rangle+1\right) / 3$ (and identical for $\left.\alpha\right)$, and since the tilt angle in the vorticity-gradient direction is effectively 0 , we find the traceless orientation tensor in the reference frame of the measurement as $\tilde{Q}_{33}=\lambda_{1}(\phi), \tilde{Q}_{22}=\mathcal{T}-\lambda_{1}(\phi) / 2$ and $\tilde{Q}_{33}=-\mathcal{T}-\lambda_{1}(\phi)$, where $\mathcal{T}=\left(\lambda_{1}(\phi) \lambda_{1}(\alpha)-\lambda_{1}(\phi)^{2}\right) / 2\left(2-\lambda_{1}(\phi)-\lambda_{1}(\alpha)\right)$. From these quantities, the full tensor $\tilde{\mathbf{S}}=(2 \tilde{\mathbf{Q}}+\mathbf{I}) / 3$ can thus be constructed and rotated into the microscopic reference frame via $\mathbf{S}=\left(\mathbf{R}^{(0, \phi)}\right)^{T}$ $\tilde{\mathbf{S}} \mathbf{R}^{(0, \phi)}$, where $R_{11}^{(0, \phi)}=R_{22}^{(0, \phi)}=\cos \theta, R_{12}^{(0, \phi)}=-R_{21}^{(0, \phi)}=\sin \theta, R_{33}^{(0, \phi)}=1$, and all other elements of the rotation tensor are zero. ${ }^{26}$ Finally, we obtain the actual order parameter $\left\langle P_{2}\right\rangle$ from the largest eigenvalue of the full tensor $\mathbf{S}$.

Materials. The rodlike bacteriophages fd wild type, fdY21M, M13k07, and M13-mini were grown in their host bacteria in LuriaBertani broth, following standard biological protocols. ${ }^{21}$ FdY21M virus is a stiff mutant of wild-type fd virus. M13k07 is a derivative of the M13 wild-type bacteriophage. All of these phages grow in Escherichia coli of the pBluescript KS(-) type, except for Pf1 virus, which is a Pseudomonas Aeruginosa phage purchased from Asla Biotech.

After purification by ultracentrifugation, a fraction of fdY21M was suspended in phosphate buffer and coated with end-functionalized monodisperse $8 \mathrm{kDa}$ polyethylen glycol (peg) in a grafting-to procedure based on click chemistry. ${ }^{60}$ The functionalized material was carefully cleansed from buffer and peg residuals by repeated ultracentrifugation and redispersion.

The peg-coated fdY $21 \mathrm{M}$ as well as bare fdY21M and all other viruses were suspended in $20 \mathrm{mM}$ Trizma base buffer solutions of deuterium oxide with $90 \mathrm{mM}$ sodium chloride, corresponding to an ionic strength of $100 \mathrm{mM}$ at $\mathrm{pH}$ 8.3. One fraction of bare fdY21M was redispersed in a $20 \mathrm{mM}$ Trizma base buffer solution of deuterium oxide, resulting in $10 \mathrm{mM}$ ionic strength at the same $\mathrm{pH}$ value. A change in ionic strength of the buffer corresponds to a change in the effective thickness of the rods. We are using effective thicknesses here, which have been determined experimentally for fd virus. ${ }^{23}$ For 100 $\mathrm{mM}$, an effective thickness of $d_{\text {eff }}=10.5 \mathrm{~nm}$ is found, which increases for $10 \mathrm{mM}$ to $d_{\text {eff }}=17 \mathrm{~nm}$, which is equal to the experimentally determined thickness of the peg-coated virus. Table 1 gives an overview of the length, $L$, and the persistence length, $L_{\mathrm{p}}$, of the various viruses.

Table 1. Length and Persistence Length of the Different Viruses

$\begin{array}{lcc}\text { material } & \text { length }(\mu \mathrm{m}) & L_{\mathrm{p}}(\mu \mathrm{m})^{61} \\ \text { fd } & 0.88 & 2.8 \pm 0.7 \\ \text { fdY21M } & 0.91 & 9.9 \pm 1.6 \\ \text { M13k07 } & 1.2 & 2.8 \pm 0.7 \\ \text { Pf1 } & 2.1 & 2.8 \pm 0.7\end{array}$

All colloidal suspensions were first prepared at a concentration just below the isotropic-nematic coexistence region and subsequently diluted to concentrations of roughly $75,50,25$, and $10 \%$ of the lowerbinodal concentration. These concentrations are well within the semidilute regime. $^{18}$

\section{RESULTS AND DISCUSSION}

In Figure 2, we plot flow curves, that is, the viscosity as a function of shear rate, for suspensions where we vary only the contour length (Figure 2a), only the thickness (Figure 2b), and only the persistence length (Figure $2 \mathrm{c}$ ). We compare data at fixed weight concentrations as the mesh size of a network of 


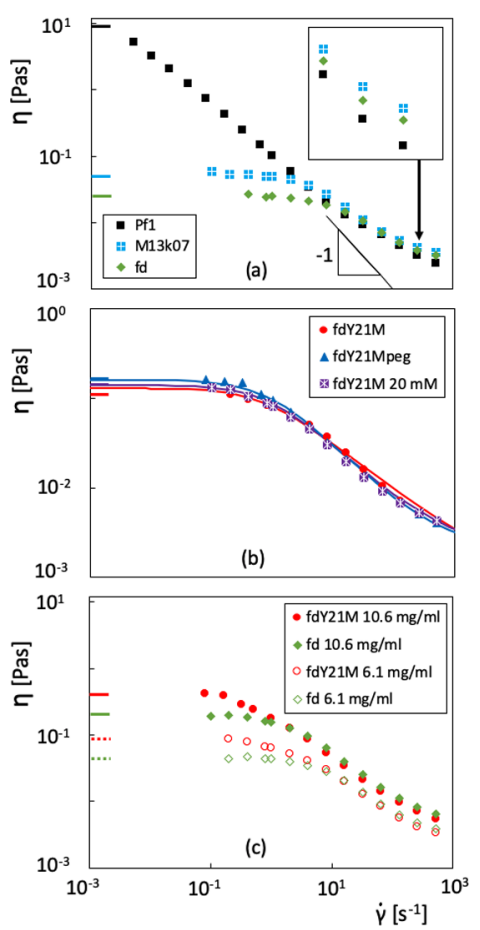

Figure 2. (a) Viscosity curves of Pf1 $(L=1.96 \mu \mathrm{m}), \operatorname{M13k} 07(L=1.2$ $\mu \mathrm{m})$, and fd $(L=0.88 \mu \mathrm{m})$ at a fixed persistence length of $L_{\mathrm{p}}=2.8 \pm$ $0.7 \mu \mathrm{m}$ and concentration of $4.8 \mathrm{mg} / \mathrm{mL}$. Inset: (magnified) viscosity at high shear rates. (b) Viscosity curves of fdY21M with different thicknesses at a concentration of $6.8 \mathrm{mg} / \mathrm{mL}$. The lines are Carreau fits with params. (Top to bottom) $a=\{1.1,1.1,1.4\} \mathrm{s}, b=\{0.7,0.65$, $0.54\}$, and $\eta_{\mathrm{s}}=8.9 \times 10^{-4} \mathrm{~Pa} \mathrm{~s}$. (c) Viscosity curves of fd wild type and fdY21M for two concentrations. The short lines at the vertical axis display the zero-shear viscosities obtained from scaling, see, e.g., Figure 3.

stiff rods, $\xi$, or tube diameter, is identical for the different particles: $\xi$ can be estimated as $\xi=L \sqrt{\nu^{*} / \nu}$, where $\nu^{*}$ is the overlap number density. ${ }^{62}$ Since $\nu^{*} \sim L^{-3}$ and $\nu \sim w[\mathrm{mg} /$ $\mathrm{mL}] / L$ (as the molecular weight is proportional to $L$ ), it follows that $\xi \sim \sqrt{w[\mathrm{mg} / \mathrm{mL}]}$. For a weight concentration of $w[\mathrm{mg} / \mathrm{mL}]=4.8 \mathrm{mg} / \mathrm{mL}$, the mesh size is equal to $0.11 \mu \mathrm{m}$, which is much smaller than the length of the viruses but is large compared to the diameter of the rods.

Figure 2a shows the flow curves for rods with varying contour lengths but identical persistence lengths. The viscosity at low shear rates strongly decreases in the order of decreasing length: an increase in length by a factor of 2 between fd virus and Pf1 causes an increase in the zero-shear viscosity by more than a decade. For the viscosities at high shear rates, the trend is partly reversed, as can be seen from the inset in Figure 2a: the flexible Pf1 now exhibits the lowest viscosity, while the other viruses retain their length-order dependence. This could be due to the fact that rods with $L_{\mathrm{p}} / L \approx 3$ can form hairpins located within planes perpendicular to the gradient direction, which strongly reduces friction, as was shown for F-actin strands in refs 63,64 . At high shear rates, there is thus a mix of shear alignment and anisotropic disentanglement that determines the viscosity.

Figure $2 \mathrm{~b}$ shows the influence of thickness on the rheological undefined for fdY $21 \mathrm{M}$, at a concentration of 6.8 $\mathrm{mg} / \mathrm{mL}$. The thickness of the virus is varied from 7 to $17 \mathrm{~nm}$ by changing the ionic strength of the suspending medium (20 and $100 \mathrm{mM}$ ) and by coating the virus with peg. As can be seen from this figure, all three viscosity curves coincide. Therefore, the thickness of the rod does not play a role, neither for small nor for large shear rates.

Figure $2 c$ shows the influence of flexibility. Here, the viscosity is plotted for fd (with $L_{\mathrm{p}} / L=3.2$ ) and fdY21M (with $\left.L_{\mathrm{p}} / L=10.9\right)$ at two different concentrations $(6.1$ and $10.6 \mathrm{mg} /$ $\mathrm{mL}$ ) in the semidilute concentration regime. Clearly, increased flexibility of particles significantly lowers the zero-shear viscosity. The length dependence of the viscosity is, however, much more pronounced than the flexibility dependence.

The slope of the viscosity curve of the longest rod in this study, Pf1, in the low shear-rate regime is significantly steeper than that of the other systems (see Figure 2a). For a slope of $\operatorname{dlog} \eta / \operatorname{dlog} \dot{\gamma} \approx-1$, one would expect to find flow instabilities. In the low to intermediate shear-rate region, Pf1 has a slope close to but not quite as steep as -1 , while all other systems display slopes of -0.5 or less. In addition, the stiffness also leads to a stronger shear thinning (see Figure $2 \mathrm{c}$ ). This points in the direction that a further increase in particle length as well as their stiffness could provoke flow instabilities such as those observed, e.g., for xanthane gum. ${ }^{65}$ It is known, however, that none of the systems under study here show flow instabilities in the long-time regime. ${ }^{27}$

Accurate values for the zero-shear viscosities $\lim _{\dot{\gamma} \rightarrow 0} \eta=\eta_{0}$ of most samples can be obtained by a fit of the flow curve to the empirical Carreau equation, ${ }^{66}\left(\eta-\eta_{\mathrm{s}}\right) /\left(\eta_{0}-\eta_{\mathrm{s}}\right)=\left[1+a \dot{\gamma}^{2}\right]^{-b}$, where $\eta_{\mathrm{s}}$ is the viscosity of the solvent, $\eta_{0}$ is the zero-shear viscosity, and the parameters $a$ and $b$ are fitting variables; see examples in Figure $2 b$. This fitting method, however, becomes ambiguous in the case of the very long rod, Pf1, where there is no clear sign of a zero-shear plateau. Therefore, we additionally employ a scaling scheme introduced by Förster et al., ${ }^{29}$ where the viscosity is plotted as a function of the scalar orientational order parameter instead of the shear rate. Figure 3a shows the unscaled viscosities as a function of the order parameter for different concentrations of fdY $21 \mathrm{M}$ in the semidilute concentration regime. Figure $3 \mathrm{~b}$ shows that the different concentrations approximately superimpose if the viscosity is scaled with an apparent zero-shear viscosity. From this scaling

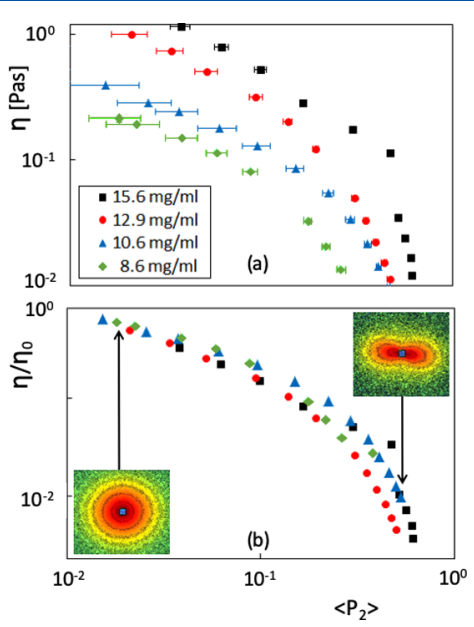

Figure 3. (a) Viscosity and (b) relative viscosity versus order parameter for different concentrations of fdY21M $\left(L=0.91 \mu \mathrm{m}, L_{\mathrm{p}}=\right.$ $9.9 \pm 1.6 \mu \mathrm{m})$. Error bars for the viscosity are inside the symbols. Insets in (b) display scattering patterns for $10.6 \mathrm{mg} / \mathrm{mL}$ at low and high shear rates. 
procedure, we obtain a second estimate for $\eta_{0}$, which compares well with the values from the Carreau fits and the viscosity close to the plateau region; see the short lines at the abscissas of Figure 2 and the direct comparison of the scaling and the Carreau fit in Figure $2 b$.

Combining the two techniques, we obtain accurate values for the zero-shear viscosities of all samples. The zero-shear viscosities for various viruses are plotted in Figure 4 as a

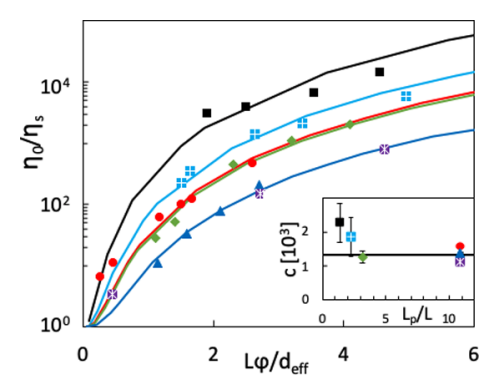

Figure 4. Zero-shear viscosity divided by the solvent viscosity as a function of the scaled volume fraction of rods. The markers are identical to those of Figure 2. Lines represent the theoretical prediction, eq 13. Inset: the fitted values of the constant $C$ in Doi's expression for the diffusion coefficient as a function of the particle stiffness. The horizontal line is the estimate from ref 55

function of concentration. The lines correspond to the prediction in eq 13, where the factor $c$ in the expression for the effective diffusion coefficient has been used as a fitting parameter. The values for the fitting parameter $c$ in Doi's expression for the effective diffusion coefficient (see the discussion just below eq 13) are shown in the inset in Figure 4 as a function of $L_{\mathrm{p}} / L$. With the exception of Pf1 (with the smallest value of $L_{\mathrm{p}} / L=1.33$ ), the values for $c$ are independent of the flexibility to within experimental error. The values of $c$ vary between $1.1 \times 10^{3}$ and $2.3 \times 10^{3}$. This is comparable to the prefactor $c=1.3 \times 10^{3}$ found from computer simulations in refs 55,56 , which value corresponds to the horizontal line in the inset in Figure 4, and a factor three lower than what was found for more flexible polymeric systems. ${ }^{67,68}$ That the zeroshear viscosities can be reasonably well described by expressions for stiff rods, including the quite flexible virus Pf1, shows that the zero-shear viscosity is relatively insensitive to flexibility as compared to the rod-length dependence.

Having established the value of $c$ in eq 8, we can proceed to describe the full shear-thinning behavior, without any further fitting parameters, using eq 9 , and the underlying nematiclike ordering quantified by the full order parameter $\left\langle P_{2}\right\rangle$ (as obtained from the full tensor $S$ ), using eq 4 . Results are shown in Figure 5a,b, for the ideal stiff rod, fdY21M, at varying concentrations and in Figure 5c,d for rods with varying lengths and fixed concentrations. Here, the shear rate is always scaled by the effective orientation-dependent rotational diffusion, see eq 8 , yielding the relevant Peclet number, $P e=\dot{\gamma} / D_{\mathrm{r}}^{\text {eff }}$. That this is the proper scaling is confirmed by the fact that for all systems, shear-thinning sets are around $P e=1$, where shear forces start to dominate rotational Brownian forces.

For the ideal stiff rod, we see that the shear-rate dependence as well as the concentration dependence of both $\eta$, Figure 5a, and $\left\langle P_{2}\right\rangle$, Figure $5 b$, are well described by our revised theory over a very large range of shear rates. In the agreement for Peclet numbers beyond unity, the good agreement is due to the effect of tube dilation, which is accounted for by the

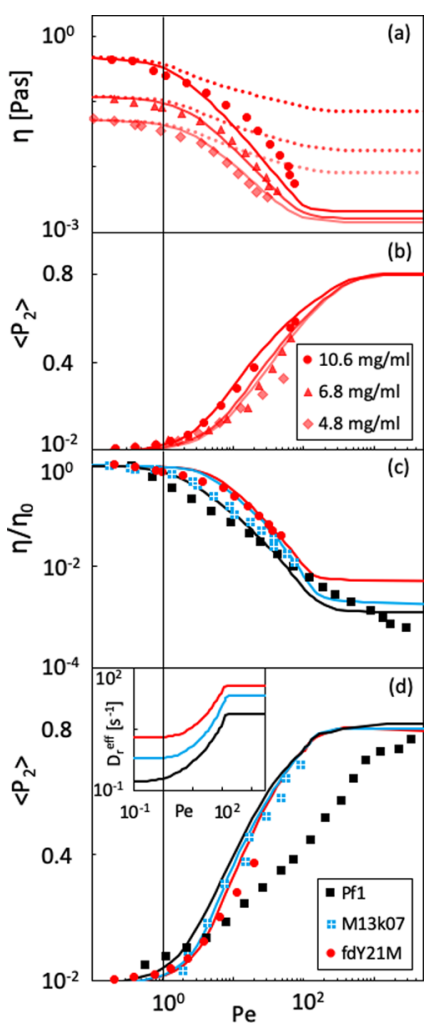

Figure 5. (a) Viscosity and (b) order parameter as a function of $P e$ for fdY21M at different concentrations. (c) Viscosity scaled by the zeroshear viscosity and $(\mathrm{d})$ order parameter as a function of the Peclet number for three rods with different lengths, Pf1 $\left(L=1.96 \mu \mathrm{m}, L_{\mathrm{p}}=\right.$ $2.8 \pm 0.7 \mu \mathrm{m}), \operatorname{M13k07}\left(L=1.2 \mu \mathrm{m}, L_{\mathrm{p}}=2.8 \pm 0.7 \mu \mathrm{m}\right)$, and fdY21M $\left(L=0.91 \mu \mathrm{m}, L_{\mathrm{p}}=9.9 \pm 1.6 \mu \mathrm{m}\right)$ for a common concentration of $\varphi=0.024$. The thick lines are theoretical predictions using eqs 8,4 , and 9, dashed lines in (a) combine eqs 4 and 9 with Doi's original diffusion coefficient. The vertical line marks $P e=1$. Inset: Rotational diffusion, eq 8 , defining the Peclet number scaling.

proposed interpolation between Doi's expression for the rotational diffusion coefficient and the free diffusion coefficient in eq 8. With increasing orientational ordering, the space available for rotation increases strongly, reaching the point of full rotatability where $D_{\mathrm{r}}^{\text {eff }}=D_{\mathrm{r}}^{0}$; see the inset of Figure $5 \mathrm{~d}$. Of course, the rods will not fully explore the created available space due to the high values of $P e$. However, if we do not allow for this strong tube dilation in our theory, the prediction of the high shear-rate viscosity deviates considerably for the measured values; see dotted lines in Figure 5a. Due to the limited shear rate that can be reached with a conventional rheometer, we could not observe the leveling off in ordering and viscosity for this system.

The length dependence of the shear-thinning process is also well captured, as can be seen in Figure $5 c$,d, where we find a masterplot when scaling $\eta$ by $\eta_{0}$. However, contrary to the relatively stiff rods, there is a significant deviation between theory and experiment in the shear-rate-dependent orientational ordering and shear thinning for the flexible Pf1, as can be seen in Figure 5d. Ordering and thinning set in at lower shear rates and are less pronounced at high shear rates. A comparatively low ratio between $L_{\mathrm{p}}$ and $L$ seems to lead to a comparatively modest increase in orientational ordering as compared to systems with lower flexibility in terms of $L_{\mathrm{p}} / L$. The reason could be the hairpin formation, as discussed above. 
Moreover, the effect of hydrodynamic friction, as suggested by Hinch and Leal, ${ }^{69}$ should not be ignored, as shown previously. ${ }^{28}$ We have discussed this issue in an earlier paper, where we treated the linear dynamic response as well as the extensional flow response of $\mathrm{fd}$ and fdY21M. Interestingly, Morse theory for the linear response, ${ }^{40,41}$ which includes flexibility, did not give as good a correspondence as the nonlinear theory which does not include flexibility. In its simplest form, the effective diffusion could be adapted ${ }^{70}$ in the $\mathrm{N}$-particle Smoluchowski equation, while in a more sophisticated version, the connection between slightly flexible rods and ideal polymer chains should be made, possibly along the lines of the work by Frey et al. ${ }^{71}$ and Broedersz and MacKintosh. ${ }^{72}$ We leave this for future studies.

\section{CONCLUSIONS}

A library of filamentous viruses probed by rheo-SANS experiments was used to assess the effect of flexibility and length of the rods on their rheological behavior in the semidilute concentration regime. The zero-shear viscosity as well as the shear-rate dependence of the viruses are contrasted with a microscopic theory based on the $N$-particle Smoluchowski equation, ${ }^{44}$ resulting in very similar expressions for the order parameter and stress tensor as derived earlier by Doi, Edwards, and Kuzuu ${ }^{38,47-49}$ (here referred to as the DEK theory). Similar to the DEK theory, dynamical correlations in the equation of motion for the order parameter are accounted for by replacing the single-rod rotational diffusion coefficient with an effective diffusion coefficient. ${ }^{47,49}$ Contrary to their theory, our theory includes the full shear-rate (as well as concentration) dependence of the tube diameter, achieving full dilation at high Peclet numbers. Together with an expression of the suspension stress in terms of the orientational ordering tensor, the presented theoretical framework describes the zeroshear viscosity, shear thinning, and the underlying nematic ordering of the ideal stiff rods without any fit parameter; see Figure 5 .

The zero-shear viscosity is found to increase with increasing length of the rodlike viruses and decreases with increasing flexibility, as can be seen in Figure 3. The length dependence of the shear viscosity is, however, much more pronounced as compared to its dependence on flexibility, especially at shear rates beyond the shear rate where shear thinning sets in. As a result, the zero-shear viscosity, and in particular the shear-rate dependence of the shear viscosity, as well as the orientational order, can be reasonably described by the theory developed for ideally stiff rods even for quite flexible rods, provided that $L_{\mathrm{p}} / L$ is larger than about 1.7. With our combined analytical and experimental effort, we can confirm the prefactor $c \approx 1.3 \times 10^{3}$, as proposed by computer simulations for Doi's rotational diffusion coefficient of crowded rod suspensions in the semidilute concentration regime. ${ }^{55,56}$

These findings together with our improved theoretical treatment allow for analytical predictions of flow properties for a large variety of systems based on rodlike particles. As such, they bear potential for use in industrial applications, where an a priori knowledge of structure-property relations, e.g., in the production process, is highly beneficial and for understanding biological processes, as stiff filaments are essential building blocks for cells.

\section{AUTHOR INFORMATION}

\section{Corresponding Authors}

*E-mail: c.lang@fz-juelich.de (C.L.).

*E-mail: joachim.kohlbrecher@psi.ch (J.K.).

*E-mail: porcar@ill.fr (L.P.).

*E-mail: a.radulescu@fz-juelich.de (A.R.).

*E-mail: k.sellinghoff@fz-juelich.de (K.S.).

*E-mail: j.k.g.dhont@fz-juelich.de (J.K.G.D.).

*E-mail: p.lettinga@fz-juelich.de (M.P.L.).

ORCID

Christian Lang: 0000-0002-8220-6568

Notes

The authors declare no competing financial interest.

\section{ACKNOWLEDGMENTS}

This research is funded by the European Union within the Horizon 2020 project under the DiStruc Marie Skłodowska Curie innovative training network; grant agreement no. 641839.

\section{REFERENCES}

(1) Wolfe, J. F.; Loo, B. H.; Arnold, F. E. Rigid-rod polymers. 2. Synthesis and thermal properties of para-aromatic polymers with 2, 6benzobisthiazole units in the main chain. Macromolecules 1981, 14, 915-920.

(2) McCulloch, I.; Heeney, M.; Bailey, C.; Geneviciusn, K.; McDonald, I.; Shkunow, M.; Sparrowe, D.; Tierney, S.; Wagner, R.; Zhang, W.; Chabinyc, M. L.; Kline, R. J.; McGehee, M. D.; Toney, M. F. Liquid-crystalline semiconducting polymers with high chargecarrier mobility. Nat. Mater. 2006, 5, 328-333.

(3) Picken, S. J.; Aerts, J.; Visser, R.; Northolt, M. G. Structure and Rheology of Aramid Solutions - X-Ray-Scattering Measurements. Macromolecules 1990, 23, 3849-3854.

(4) Monteiro, S. N.; Lima, E. P.; Louro, L. H. L.; Da Silva, L. C.; Drelich, J. W. Unlocking Function of Aramid Fibers in Multilayered Ballistic Armor. Metall. Mater. Trans. A 2015, 46, 37-40.

(5) Vigolo, B.; Penicaud, A.; Coulon, C.; Sauder, C.; Pailler, R.; Journet, C.; Bernier, P.; Poulin, P. Macroscopic fibers and ribbons of oriented carbon nanotubes. Science 2000, 290, 1331-1334.

(6) Kouwer, P. H. J.; Koepf, M.; Le Sage, V. A. A.; Jaspers, M.; van Buul, A. M.; Eksteen-Akeroyd, Z. H.; Woltinge, T.; Schwartz, E.; Kitto, H. J.; Hoogenboom, R.; Picken, S. J.; Nolte, R. J. M.; Mendes, E.; Rowan, A. E. Responsive biomimetic networks from polyisocyanopeptide hydrogels. Nature 2013, 493, 651-655.

(7) Tolstoguzov, V. Phase behaviour of macromolecular components in biological and food systems. Nahrung 2000, 44, 299-308.

(8) Khan, T.; Park, K.; Kwon, J.-H. Functional biopolymers produced by biochemical technology considering applications in food engineering. Korean J. Chem. Eng. 2007, 24, 816-826.

(9) van der Linden, E.; Venema, P. Self-assembly and aggregation of proteins. Curr. Opin. Colloid Interface Sci. 2007, 12, 158-165.

(10) Araki, J. Electrostatic or steric?-preparations and characterizations of well-dispersed systems containing rod-like nanowhiskers of crystalline polysaccharides. Soft Matter 2013, 9, 4125-4141J.

(11) Cherny, I.; Gazit, E. Amyloids: not only pathological agents but also ordered nanomaterials. Angew. Chem., Int. Ed. 2008, 47, 40624069.

(12) Olson, M. F.; Sahai, E. The actin cytoskeleton in cancer cell motility. Clin. Exp. Metastasis 2009, 26, 273-287.

(13) Maji, S. K.; Perrin, M. H.; Sawaya, M.; Jessberger, S.; Vadodaria, K.; Rissmann, R. A.; Singru, P. S.; Nilsson, K. P. R.; Simon, R.; Schubert, D.; Eisenberg, D.; Rivier, J.; Sawchenko, P.; Vale, W.; Riek, R. Functional amyloids as natural storage of peptide hormones in pituitary secretory granules. Science 2009, 325, 328-332.

(14) Stricker, J.; Falzone, T.; Gardel, M. L. Mechanics of the F-actin cytoskeleton. J. Biomech. 2010, 43, 9-14. 
(15) Djalili-Moghaddam, M.; Toll, S. Fibre suspension rheology: effect of concentration, aspect ratio and fibre size. Rheol. Acta 2006, $45,315-320$.

(16) Boluk, Y.; Lahiji, R.; Zhao, L.; McDermott, M. T. Suspension viscosities and shape parameter of cellulose nanocrystals (CNC). Colloids Surf., A 2011, 377, 297-303.

(17) Tanaka, R.; Saito, T.; Isihi, D.; Isogai, A. Determination of nanocellulose fibril length by shear viscosity measurement. Cellulose 2014, 21, 1581-1589.

(18) Graf, C.; Kramer, H.; Deggelmann, M.; Hagenbuechle, M.; Johner, C.; Johner, C. M.; Weber, R. Rheological properties of suspensions of interacting rodlike FD-virus particles. J. Chem. Phys. 1993, 98, 4920.

(19) Wierenga, A. M.; Philipse, A. P. Low-shear viscosity of isotropic dispersions of (Brownian) rods and fibres. A review of theory and experiments. Colloids Surf., A 1998, 137, 355-372.

(20) Dogic, Z. Filamentous phages as a model system in soft matter physics. Front. Microbiol. 2016, 7, 1013.

(21) Sambrook, J.; Fritsch, E. F.; T, M. Molecular Cloning: a Laboratory Manual; Cold Spring Harbor Laboatory Press: Cold Spring Harbor, New York, 1989.

(22) Grelet, E.; Rana, R. From soft to hard rod behavior in liquid crystalline suspensions of sterically stabilized colloidal filamentous particles. Soft Matter 2016, 12, 4621-4627.

(23) Dogic, Z.; Fraden, S. Ordered phases of filamentous viruses. Curr. Opin. Colloid Interface Sci. 2006, 11, 47-55.

(24) Lettinga, M. P.; Dogic, Z.; Wang, H.; Vermant, J. Flow behavior of colloidal rodlike viruses in the nematic phase. Langmuir 2005, 21, 8048-8057.

(25) Ripoll, M.; Holmqvist, P.; Winkler, R. G.; Gompper, G.; Dhont, J. K.; Lettinga, M. P. Attractive colloidal rods in shear flow. Phys. Rev. Lett. 2008, 101, No. 168302.

(26) Lang, C.; Kohlbrecher, J.; Porcar, L.; Lettinga, M. P. The connection between biaxial orientation and shear thinning for quasiideal rods. Polymers 2016, 8, 291.

(27) Lang, C.; Porcar, L.; Kriegs, H.; Lettinga, M. P. A quest for shear banding in ideal and non ideal colloidal rods. J. Phys. D.: Appl. Phys. 2019, 52, No. 074003.

(28) Lang, C.; Hendricks, J.; Zhang, Z.; Reddy, N. K.; Rothstein, J. P.; Lettinga, M. P.; Vermant, J.; Clasen, C. Effects of particle stiffness on the extensional rheology of model rod-like nanoparticle suspensions. Soft Matter 2019, 15, 833-841.

(29) Förster, S.; Konrad, M.; Lindner, P. Shear thinning and orientational ordering of wormlike micelles. Phys. Rev. Lett. 2005, 94, No. 017803.

(30) Liberatore, M. W.; Nettesheim, F.; Wagner, N.; Porcar, L. Spatially resolved small-angle neutron scattering in the 1-2 plane: A study of shear-induced phase-separating wormlike micelles. Phys. Rev. E 2006, 73, No. 020504.

(31) Liberatore, M. W.; Nettesheim, F.; Vasquez, P. A.; Helgeson, M. E.; Wagner, N. J.; Kaler, E. W.; Cook, L. P.; Porcar, L.; Hu, Y. T. Microstructure and shear rheology of entangled wormlike micelles in solution. J. Rheol. 2009, 53, 441.

(32) Onsager, L. The effects of shape on the interaction of colloidal particles. Ann. N. Y. Acad. Sci. 1949, 51, 627-659.

(33) Grelet, E. Hexagonal Order in Crystalline and Columnar Phases of Hard Rods. Phys. Rev. Lett. 2008, 100, No. 168301.

(34) Odijk, T. The statistics and dynamics of confined or entangled stiff polymers. Macromolecules 1983, 16, 1340-1344.

(35) Odijk, T. Theory of lyotropic polymer liquid crystals. Macromolecules 1986, 19, 2313-2329.

(36) Vroege, G. J.; Lekkerkerker, H. N. W. Phase transitions in lyotropic colloidal and polymer liquid crystals. Rep. Prog. Phys. 1992, $55,1241-1309$.

(37) Hess, S. Fokker-Planck-equation approach to flow alignment in liquid crystals. Z. Naturforsch., A 1976, 31, 1034-1037.

(38) Doi, M. Molecular dynamics and rheological properties of concentrated solutions of rodlike polymers in isotropic and liquid crystalline phases. J. Polym. Sci., Polym. Phys. Ed. 1981, 19, 229-243.
(39) Ranganathan, S.; Advani, S. G. Fiber-fiber interactions in homogeneous flows of nondilute suspensions. J. Rheol. 1991, 35, 1499.

(40) Morse, D. C. Viscoelasticity of concentrated isotropic solutions of semiflexible polymers. 1. Model and stress tensor. Macromolecules 1998, 31, 7030-7043.

(41) Morse, D. C. Viscoelasticity of concentrated isotropic solutions of semiflexible polymers. 2. Linear response. Macromolecules 1998, 31, 7044-7067.

(42) Morse, D. C. Viscoelasticity of concentrated isotropic solutions of semiflexible polymers. 3. nonlinear rheology. Macromolecules 1999, 32, 5934-5943.

(43) Switzer, L. H.; Klingenberg, D. J. Rheology of sheared flexible fiber suspensions via fiber-level simulations. J. Rheol. 2003, 47, 759.

(44) Dhont, J. K. G.; Briels, W. J. Viscoelasticity of suspensions of long, rigid rods. Colloids Surf., A 2003, 213, 131-156.

(45) Zirnsak, M. A.; Boger, B. V.; Tirtaatmadja, V. Steady shear and dynamic rheological properties of xanthan gum solutions in viscous solvents. J. Rheol. 1999, 43, 627.

(46) Hobbie, E. K.; Wang, H.; Kim, H.; Han, C. C.; Grulke, E. A.; Obrzut, J. Optical measurements of structure and orientation in sheared carbon-nanotube suspensions. Rev. Sci. Instrum. 2003, 74, $1244-1250$

(47) Doi, M.; Edwards, S. F. Dynamics of rod-like macromolecules in concentrated solution Part 1. J. Chem. Soc., Faraday Trans. 2 1978, $74,560-570$.

(48) Doi, M.; Edwards, S. F. The Theory of Polymer Dynamics; Oxford University Press: New York, 1986.

(49) Kuzuu, N.; Doi, M. Constitutive equation for nematic liquid crystals under weak velocity gradient derived from a molecular kinetic equation. J. Phys. Soc. Jpn. 1983, 52, 3486-3494.

(50) Smoluchowski, M. V. Ueber Brownsche Molekularbewegung unter Einwirkung aeußerer Kraefte und deren Zusammenhang mit der verallgemeinerten Diffusionsgleichung. Ann. Phys. 1916, 353, 11031112 .

(51) Onsager, L. Theories of Concentrated Electrolytes. Chem. Rev. 1933, 13, 73-89.

(52) Replacing the bare diffusion coefficient by the effective coefficient only in the term $\sim(L / d) \varphi$ leads to a thermodynamic inconsistency. When this is done, eq 3 reads, in the absence of flow

$$
\frac{\mathrm{d} \mathbf{S}}{\mathrm{d} t}=-6\left[D_{\mathrm{r}}^{0}\left(\mathbf{S}-\frac{1}{3} \mathbf{I}\right)+D_{\mathrm{r}}^{\text {eff }} \frac{L}{d} \varphi\left(\mathbf{S}^{(4)}: \mathbf{S}-\mathbf{S} \cdot \mathbf{S}\right)\right]
$$

Consider now the concentration where the isotropic state becomes unstable, in the absence of flow. Writing $\mathbf{S}=(1 / 3) \hat{\mathbf{I}}+\delta \mathbf{S}$, and linearisation with respect to $\delta \mathrm{S}$ leads to

$$
\frac{\mathrm{d}}{\mathrm{d} t} \delta \mathbf{S}=-6\left[D_{\mathrm{r}}^{0}-\frac{1}{5} \frac{L}{d} \varphi D_{\mathrm{r}}^{\mathrm{eff}}\right] \delta \mathbf{S}
$$

It follows from this equation of motion that the isotropic state is unstable for concentrations for which $(L / 5 d) \varphi>D_{\mathrm{r}}^{0} / D_{\mathrm{r}}^{\text {eff. }}$. Since phase stability can not depend on the dynamics of the system, we are forced to chose both rotational diffusion coefficients equal, so that the isotropic-to-nematic spinodal is located at $(L / 5 d) \varphi>1$. As discussed in the main text, the interpretation is that each rod experiences an effective medium, which also affects the otherwise ideal relaxation contribution (the term $\sim(S-(1 / 3) \hat{\mathbf{I}}))$.

(53) Kröger, M.; Ammar, A.; Chinesta, F. Consistent closure schemes for statistical models of anisotropic fluids. J. Non-Newtonian Fluid Mech. 2008, 149, 40-55.

(54) Takada, Y.; Sato, T.; Teramoto, A. Dynamics of stiff-chain polymers in isotropic solution. 2. Viscosity of aqueous solutions of xanthan, a rigid double-helical polysaccharide. Macromolecules 1991, 24, 6215-6219.

(55) Teraoka, I.; Ookubo, N.; Hayakawa, R. Molecular theory on the entanglement effect of rodlike polymers. Phys. Rev. Lett. 1985, 55, $2712-2715$. 
(56) Tao, Y. G.; den Otter, W. K.; Dhont, J. K. G.; Briels, W. J. Isotropic-nematic spinodals of rigid long thin rodlike colloids by event-driven Brownian dynamics simulations. J. Chem. Phys. 2006, 124, No. 134906.

(57) Dhont, J. K. G.; Briels, W. J. Stresses in inhomogeneous suspensions. J. Chem. Phys. 2002, 117, 3992-3999.

(58) Hayter, J. B.; Penfold, J. Use of viscous shear alignment to study anisotropic micellar structure by small angle neutron scattering. $J$. Phys. Chem. A 1984, 88, 4589-4598.

(59) Maier, W.; Saupe, A. Eine einfache molekular-statistische Theorie der nematischen kristallinfluessigen Phase. Teil 1. Z. Naturforsch., A 1959, 14, 882-889.

(60) Zhang, Z.; Buitenhuis, J.; Cukkemane, A.; Brocker, M.; Bott, M.; Dhont, J. K. G. Charge reversal of the rodlike colloidal fd virus through surface chemical modification. Langmuir 2010, 26, 1059310599 .

(61) Barry, E.; Beller, D.; Dogic, Z. A model liquid crystalline system based on rodlike viruses with variable chirality and persistence length. Soft Matter 2009, 5, 2563-2570.

(62) de Gennes, P.-G.; Pincus, P.; Velasco, R. M.; Brochard, F. Remarks on polyelectrolyte conformation. J. Phys. France 1976, 37, 1461.

(63) Kirchenbuechler, I.; Guu, D.; Kurniawan, N. A.; Koenderink, G. H.; Lettinga, M. P. Direct visualization of flow-induced conformational transitions of single actin filaments in entangled solutions. Nat. Commun. 2014, 5, No. 5060.

(64) Huber, B.; Harasim, M.; Wunderlicha, B.; Kroeger, M.; Bausch, A. R. Microscopic origin of the non-Newtonian viscosity of semiflexible polymer solutions in the semidilute regime. ACS Macro Lett. 2014, 3, 136-140.

(65) Tang, H.; Kochetkova, T.; Kriegs, H.; Dhont, J. K. G.; Lettinga, M. P. Shear-banding in entangled xanthan solutions: tunable transition from sharp to broad shear-band interfaces. Soft Matter 2018, 14, 826.

(66) Carreau, P. J. Rheological equations from molecular network theories. Trans. Soc. Rheol. 1972, 16, 99-127.

(67) Sato, T.; Teramoto, A. Dynamics of Stiff-Chain Polymers in Isotropic Solution - Zero-Shear Viscosity of Rodlike Polymers. Macromolecules 1991, 24, 193-196.

(68) Petekidis, G.; Vlassopoulos, D.; Fytas, G.; Fleischer, G.; Wegner, G. Dynamics of wormlike polymers in solution: Self-diffusion and zero-shear viscosity. Macromolecules 2000, 33, 9630-9640.

(69) Hinch, E. J.; Leal, L. G. The effect of Brownian motion on the rheological properties of a suspension of non-spherical particles. J. Fluid Mech. 1972, 52, 683-712.

(70) Sato, T.; Takada, Y.; Teramoto, A. Dynamics of Stiff-Chain Polymers in Isotropic Solution. 3. Flexibility Effect. Macromolecules 1991, 24, 6220-6226.

(71) Frey, E.; Kroy, K.; Wilhelm, J. Viscoelasticity of biopolymer networks and statistical mechanics of semiflexible polymers. Adv. Struct. Biol. 1999, 5, 135-168.

(72) Broedersz, C. P.; MacKintosh, F. C. Modeling semiflexible polymer networks. Rev. Mod. Phys. 2014, 86, 995-1036. 